\title{
Invasive pericranial nerve interventions
}

\author{
Anna Ambrosini' and Jean Schoenen ${ }^{2}$
}

Cephalalgia

0 (0) $\mathrm{I}-\mathrm{I} 4$

(C) International Headache Society 2016

Reprints and permissions:

sagepub.co.uk/journalsPermissions.nav DOI: $10.1177 / 0333102416639515$

cep.sagepub.com

\begin{abstract}
Background: In many patients suffering from primary headaches, the available pharmacological and behavioural treatments are not satisfactory. This is a review of (minimally) invasive interventions targeting pericranial nerves that could be effective in refractory patients.

Methods: The interventions we will cover have in common pericranial nerves as targets, but are distinct according to their rationale, modality and invasiveness. They range from nerve blocks/infiltrations to the percutaneous implantation of neurostimulators and surgical decompression procedures. We have critically analysed the published data (PubMed) on their effectiveness and tolerability.

Results and conclusions: There is clear evidence for a preventative effect of suboccipital injections of local anaesthetics and/or steroids in cluster headache, while evidence for such an effect is weak in migraine. Percutaneous occipital nerve stimulation (ONS) provides significant long-term relief in more than half of drug-resistant chronic cluster headache patients, but no sham-controlled trial has tested this. The evidence that ONS has lasting beneficial effects in chronic migraine is at best equivocal. Suboccipital infiltrations are quasi-devoid of side effects, while ONS is endowed with numerous, though reversible, adverse events. Claims that surgical decompression of multiple pericranial nerves is effective in migraine are not substantiated by large, rigorous, randomized and sham-controlled trials.
\end{abstract}

\section{Keywords}

Migraine, cluster headache, treatment, surgical decompression, percutaneous neurostimulation, nerve blocks

Date received: 3 November 2015; revised: 22 February 2016; accepted: 26 February 2016

\section{Introduction}

Primary headaches altogether represent the most common neurological disorders and are usually associated with disability, decreased quality of life and financial costs both for the affected individual and for society. Among primary headaches, migraine and cluster headaches are the ones that more often affect quality of life, especially their chronic forms, which are frequently associated with acute medication overuse and low responsiveness to preventative pharmacological treatments. Approximately $11 \%$ of all migraine patients and approximately $1 \%$ of chronic cluster headache $(\mathrm{CCH})$ patients become refractory to all available pharmacological treatments (1).

The management of migraine and cluster headache is multifaceted. Besides advice on lifestyle, the management of cluster headache is based on acute interventions in order to alleviate the attack and preventative treatments in order to reduce attack frequency and disability. However, the efficacy of preventive drugs is limited in many patients, and most of these pharmacological treatments can have cumbersome adverse effects
(2). Alternative treatments have thus been used for some time, but they have received increasing attention only recently because of the lack of new advances in pharmacotherapy.

Migraine and cluster headache are considered to be central neurovascular disorders. The headache is likely generated in the trigeminovascular system that cortical (migraine) or subcortical (cluster headache) dysfunctions are thought to activate (3). It was recently shown that trigeminovascular meningeal afferents project through the skull (4) and that activation of these extracranial afferents in rats causes the release of calcitonin gene related peptide (CGRP) from the dura,

\footnotetext{
'IRCCS NEUROMED, Pozzilli (Isernia), Italy

${ }^{2}$ Headache Research Unit, University of Liège, Citadelle Hospital, Belgium
}

\section{Corresponding author:}

Anna Ambrosini, Headache Unit, IRCCS NEUROMED, Via Atinense, 18, 86077, Pozzilli (Isernia), Italy.

Email: anna.ambrosini@neuromed.it 
suggesting that extracranial noxious signals may influence meningeal nociception (5). Despite the scarcity of data favouring a role for pericranial nerves in primary headaches, these findings may offer a rationale for the various therapeutic interventions based on pericranial nerves that have been used in refractory patients for some time. Many patients indeed state that their pain is localised to the surface of the head or neck, which is in close anatomical relationship with branches of the pericranial nerves. Although these superficial pain locations were likely to represent referred pain from the visceral part of the ophthalmic division of the trigeminal nerve, they led to surgical interventions on superficial cranial nerves for the treatment of migraine and other headache types by as early as the first half of the 20th century. Renowned physicians such as Cushing, Penfield or Rowbotham pioneered subtotal ophthalmic nerve or cervical root rhizotomies, sections of supraorbital, supratrochlear or occipital nerves, excisions of the stellate ganglion or pericarotid sympathectomies, and even craniotomies (6). Various invasive lesional procedures have also been performed over time in cluster headache, targeting the trigeminal or cranial parasympathetic pathways and using radiofrequency lesions, glycerol injections or balloon compressions of the Gasserian ganglion, Gamma Knife surgery or section of the trigeminal root, trigeminal tractotomy, lesions of the nervus intermedius or greater superficial petrosal nerve, blockade or radiofrequency lesions of the sphenopalatine ganglion and microvascular decompression of the trigeminal nerve combined with nervus intermedius section (7). Although spectacular results were reported in some patients, most of them had a mixture of different headache types and few showed satisfactory long-term results, despite the mutilating character of the procedures (6).

We will focus here on some of the invasive interventions that are used to treat migraine and cluster headache and are supposed to act on pericranial nerve branches. We have schematically ordered them according to their increasing invasiveness from infiltrations/ blocks to percutaneous neurostimulations and surgical decompressions.

\section{Blocks and infiltrations}

Peripheral nerve blocks have been used for some time in order to treat headaches (8). In most studies they targeted the greater occipital nerve (GON) because of the known anatomo-physiological convergence of $\mathrm{C} 2$ dermatoma and trigeminovascular afferents in the spinal trigeminal nucleus that underlies referred pain from the neck to the orbitofrontal regions innervated by the ophthalmic nerve (9). Local anaesthetics are commonly injected alone or in combination with corticosteroids.
The procedure is minimally invasive, inexpensive, safe and it can be performed on an outpatient basis.

\section{Migraine}

The most relevant studies are summarized in Table 1 (10-14). Five controlled trials are available, but unfortunately no standardised methods were used. The selection criteria of patients varied between studies (some had fixed unilateral headache, others not) and so did the timing of the procedure (ictal or inter-ictal), the technique of infiltrations or blocks (unilateral or bilateral, associated blocks of other pericranial nerves or trigger point injections, one or more interventions), the compounds used for the blocks (local anaesthetics alone or combined with different types and dosages of steroids) and the outcome measures (number of headache-free days, percentage reduction of headache days or attacks, non-standardised pain indices). A comparison of results between studies is therefore challenging.

Overall, a complete or partial benefit for migraine prevention was reported in $48-100 \%$ of adult migraineurs, lasting from a few days to several months. A retrospective study also found a partial benefit $(<35 \%)$ from GON injections in paediatric chronic migraineurs (15). In none of the controlled studies on GON blocks was the addition of steroids found to be superior to the injection of a local anaesthetic alone, commonly used as placebo. In one controlled singleblinded study, the benefit was quite modest (10). Similarly, in a placebo-controlled randomized doubleblinded trial performed in episodic $(n=54)$ and chronic $(n=9)$ migraineurs, the block with steroids showed a non-significant percentage of $>50 \%$ responders $(\sim 30 \%)$ (11). In a third randomised trial in episodic migraine patients, $1.0 \mathrm{~mL}$ of lidocaine $2 \%$ injected suboccipitally induced a similar decrease of pain severity and frequency for up to 8 weeks, whether it was combined with $0.5 \mathrm{~mL}$ saline (control group: $n=24$ ) or with $0.5 \mathrm{~mL}$ triamcinolone $(n=24)(12)$.

Two recent double-blind placebo-controlled studies comparing GON injections of saline (placebo) with $0.5 \%$ bupivacaine (verum) found that the latter provided partial benefit in migraine patients. In the first study, chronic migraine patients received four weekly injections (13). The number of headache days decreased from $16.9 \pm 5.7$ to $13.2 \pm 6.7$ in the placebo group $(p=0.035)$, but from $18.1 \pm 5.3$ to $8.8 \pm 4.8(p<0.001)$ in verum-treated patients, and the superior effect of bupivacaine was confirmed in an open 2-month extension study. In the second study, patients suffering from chronic refractory migraine had a single ultrasoundguided GON block of saline or bupivacaine $0.5 \%$. Four weeks later, the average pain intensity score on the injected side was significantly decreased in the 


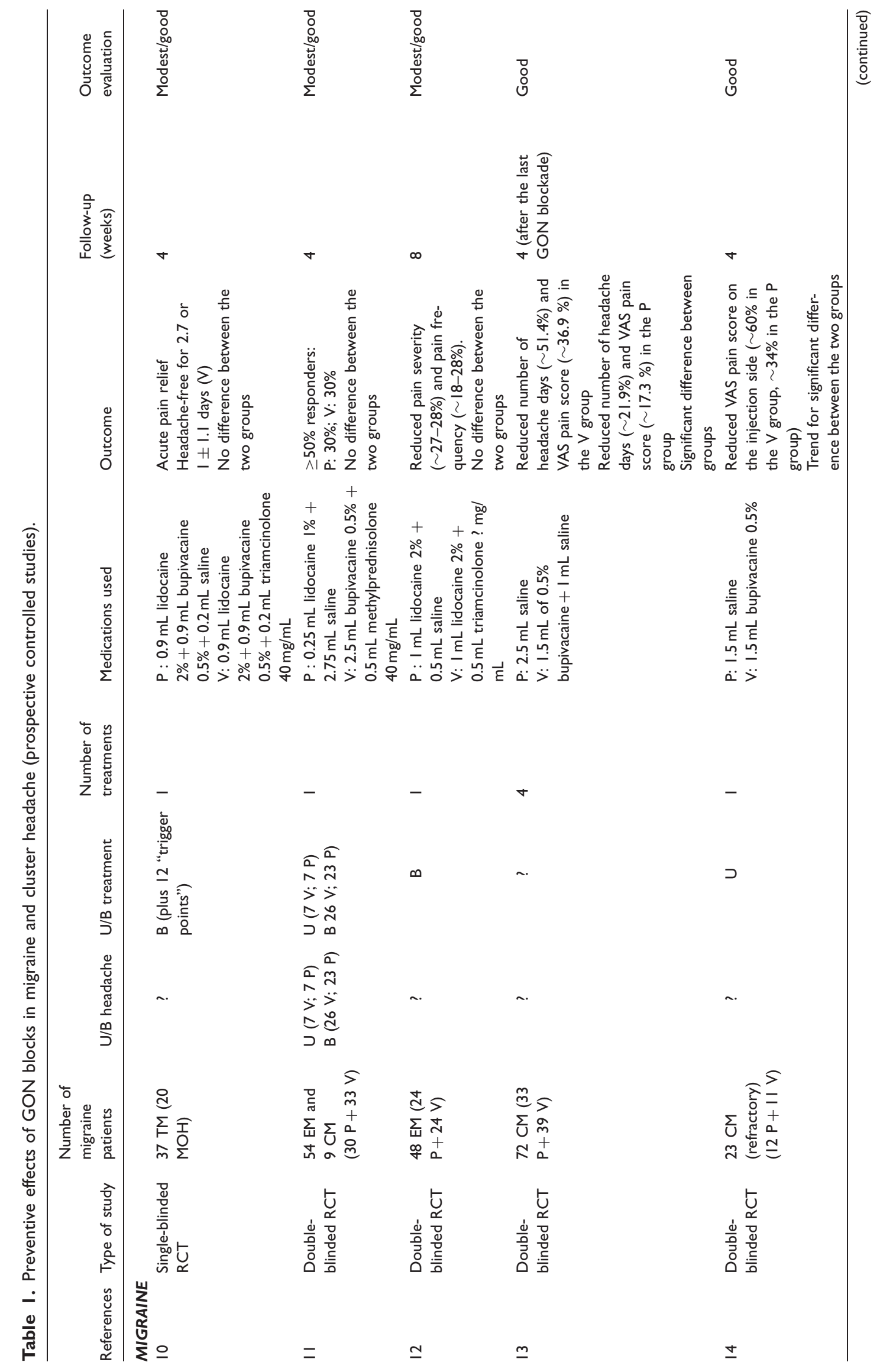




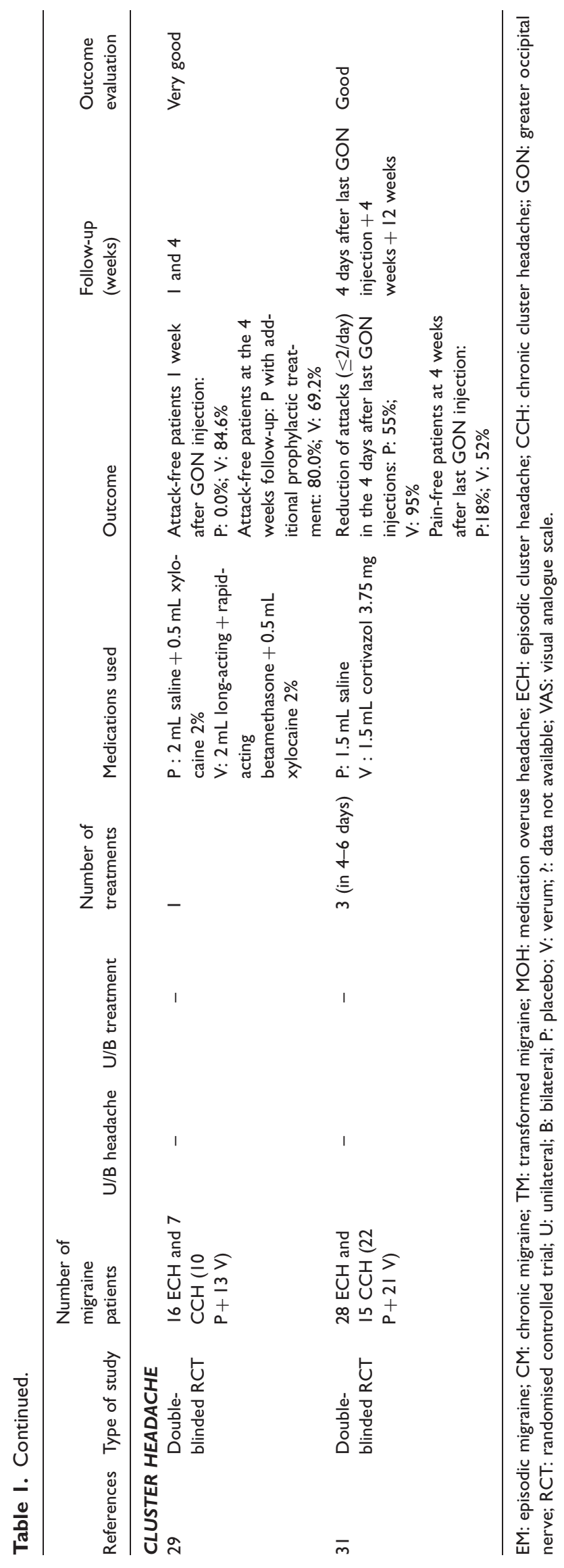


verum $(n=11 ; p=0.003)$ but not in the placebo group $(n=12 ; p=0.110)(14)$.

Medication overuse was not associated with an increased risk of failure of GON blocks in two studies $(16,17)$, but it tripled this risk in another study (18). The presence of GON palpation tenderness was associated with better outcome in one study (16), but not in two others $(12,17)$. The effect of GON blocks on headache frequency and intensity does not predict the success of GON neurostimulation in chronic migraineurs (19).

GON blocks were also tested as a symptomatic treatment for migraine attacks. In 14 patients who received combined lidocaine blocks of the greater occipital nerve and supraorbital nerve (SON), pain reduction after 30 minutes was almost significant, but $50 \%$ of patients did not respond at all (20). By contrast, in an open study in which a GON block with $1 \mathrm{cc}$ of a 50/50 mixture of $2 \%$ lidocaine and $0.5 \%$ bupivacaine was administered to patients with unilateral episodic or chronic migraine and brush allodynia, headache intensity decreased on average by $46.8 \%$ in $89.5 \%$ of patients after 20 minutes and ipsilateral allodynia decreased by $65.7 \%$ in all patients (21). The same group reported that the procedure produced $64 \%$ and $75 \%$ reductions of pain and allodynia scores, respectively, after 5 minutes and that the benefit lasted on average for 4 days, both in episodic and chronic migraineurs (22). In a single case report, GON infiltration with $3 \mathrm{~mL}$ of $0.25 \%$ bupivacaine and $1 \mathrm{~mL}$ of $40 \mathrm{mg} / \mathrm{mL}$ triamcinolone induced partial resolution of aura symptoms and complete disappearance of the headache after a few minutes in a patient with basilar-type migraine who was treated during an attack (23).

SON blocks have rarely been studied without concomitant GON blocks, so that their proper efficacy is difficult to assess. The combination of GON and SON blocks with $0.5-1.5 \mathrm{~mL}$ of lidocaine $(20 \mathrm{mg} / \mathrm{mL}$ and $12.5 \mu \mathrm{g} / \mathrm{mL}$ adrenaline) had no beneficial effect in one study (20). By contrast, in an open trial comparing SON and GON blocks alone and their combination, SON blocks alone reduced headache frequency by $50 \%$ in $68.75 \%$ of patients after 1 month and in $75 \%$ of patients after 6 months (24). A further study reported that three consecutive bilateral SON and infraorbital nerve blocks with $1.5 \mathrm{~mL}$ of $1 \%$ lidocaine in episodic migraine patients were able to decrease significantly the mean headache frequency and migraine disability assessment (MIDAS) score (25).

In a recent randomized, double-blind, placebocontrolled trial, repetitive $0.5 \%$ bupivacaine blockades of the sphenopalatine ganglion with the Tx $360^{\circledR}$ device twice a week for 6 weeks were compared to saline injections in chronic migraine patients (26). Slight but non-significant reductions in headache days and average pain scores were found in the bupivacaine group.

\section{Cluster headache}

A large number of open studies - reviewed in Leroux and Ducros (27) - found that GON blocks with steroids were beneficial as a transitional treatment during active bouts in episodic cluster headache $(\mathrm{ECH})$ and in $\mathrm{CCH}$ patients who had an insufficient response to classical oral preventative drugs, while GON blocks solely with local anaesthetics were ineffective (28-30). At present, only two randomized, placebo-controlled studies are available on GON blockade in cluster headache. In the first study, $\mathrm{CCH}(n=7)$ and active ECH $(n=16)$ patients were included (29). The verum group $(n=13)$ received a single GON infiltration on the side of attacks with a mixture containing a long-acting salt and a rapid-acting salt of betamethasone (dipropionate $12.46 \mathrm{mg}$ and disodium phosphate $5.26 \mathrm{mg}$, respectively) mixed with $0.5 \mathrm{~mL}$ of lidocaine $2 \%$, while the placebo-treated group received physiological saline and $0.5 \mathrm{~mL}$ of lidocaine $2 \%$. The stringent outcome measure of sustained attack-free state within 72 hours for 4 weeks was fulfilled by $61.5 \%$ of steroid-injected patients, contrasting with none of the placebo-treated patients. A total of $85 \%$ of steroid-injected patients had a remission after 72 hours for at least 1 week. Only three patients out of the 11 who initially responded had recurrence of attacks within the 4-week duration of the trial. One additional patient was considered to be a non-responder because his attacks did not disappear within 72 hours of the injection, but he became asymptomatic 1 day later. Five out of eight responders remained in total remission for at least 6 months. Among the ten patients who were injected with physiological saline-lidocaine, none was attack-free after 1 week, and all of them needed additional prophylactic treatment.

The second randomised controlled trial (RCT) included 43 patients $(28 \mathrm{ECH}, 15 \mathrm{CCH})$ who received three unilateral suboccipital injections 48-72 hours apart of either cortivazol $3.75 \mathrm{mg}$ (verum group, $n=21$ ) or physiological saline (placebo group, $n=22$ ) (31). In the first 2 weeks, patients in the cortivazol-treated group had on average a significant reduction of attack frequency $(p=0.004)$, and there was a trend for a higher percentage of patients with a $>50 \%$ reduction of attacks compared to the placebo group ( $86 \%$ vs. $59 \% ; p=0.064)$. At day 30 after the injections, $76 \%$ of cortivazol-treated and $59 \%$ of saline-treated patients were in remission.

\section{Comments}

Most observational studies suggest that GON infiltrations with local anaesthetics or steroids and, to a lesser degree, SON blocks may be beneficial in some 
migraine patients, but further studies are needed in order to determine in which subgroups this beneficial effect occurs. By contrast, in three out of five controlled trials, the effect of GON blockade was marginal. Given the available evidence, GON blocks cannot be considered a first-line treatment in migraine. They are at best useful as an add-on therapy for the treatment of status migrainosus or for short-term prophylaxis in chronic migraine with medication overuse, with the aim of reducing the consumption of symptomatic drugs until preventative treatments become effective (32).

In two RCTs, GON infiltrations with steroids were clearly superior to physiological saline (31) or a local anaesthetic alone (29) in terms of interrupting a bout of cluster headache. This contrasts with migraine, for which some studies also reported a positive effect of the injection of a sole local anaesthetic.

Overall, GON blocks are well tolerated. Adverse effects are rare and minor, and include dizziness, local pain and vaso-vagal pre-syncope. Alopecia was reported in some patients who were injected superficially with triamcinolone; this can be avoided completely by injecting steroids in a deeper location close to the anatomical area where the GON has a lateromedial trajectory before becoming superficial next to the midline (33).

\section{Invasive peripheral nerve neurostimulation}

Electrical stimulation of peripheral nerves (PNS) is a well-known and efficacious way to relieve pain within the territory of the stimulated nerve. Its effect is thought to be due to activation of afferent $A \beta$ fibres and subsequent gate control mechanisms in the spinal cord dorsal horn and/or activation of descending supraspinal pain controls from the periaqueductal grey and rostral ventromedial medulla $(34,35)$.* Visceral trigeminovascular afferents converge with cervical (C2) and somatic ophthalmic nerve afferents on second-order nociceptors in the spinal trigeminal nucleus (36). There is thus, in theory, a rationale for stimulation of the GON and/or the SON in migraine and cluster headache.

\section{Migraine}

Invasive PNS was studied as a preventative therapy quasi-exclusively in patients suffering from drugresistant chronic migraine.

In addition to a small, randomized open trial, only three randomized sham-controlled trials have been published, two in extenso and one in abstract form (37-40).
In the open-label trial, 29 patients who did not respond to at least two prophylactic treatments and had benefited from a preliminary temporary occipital nerve stimulation (ONS) system were ameliorated when the ONS device was switched on as compared to when it was switched off, and these benefits were confirmed in a 1-year follow-up (37). In the ONSTIM trial that included 66 patients with a 12-week follow-up, 39\% of patients who received the active ONS had at least a $50 \%$ decrease in headache frequency and headache intensity, while there were no improvements in the sham-stimulated and medically treated groups (Table 2) (38). Unfortunately, ONSTIM was not powered to demonstrate efficacy, but rather feasibility and safety. By contrast, in the sham-controlled PRISM study, in which 125 patients with drug-resistant chronic migraine with or without medication overuse were enrolled, there was no superiority of active ONS over sham stimulation (39). The hitherto largest RCT of ONS in 157 chronic migraine patients failed to reach the primary endpoint (at least $50 \%$ reduction in mean daily headache intensity), but a higher percentage of patients in the active than in the sham arm achieved a $30 \%$ reduction in mean headache days $(p<0.05)$ and a decrease in migraine-related disability score (MIDAS) $(p<0.01)(40)$. After the 3-month randomised phase, an open-label phase of 40 weeks showed a significant 6.7-day reduction in monthly headache days in the intention-to-treat group and a 7.7-day reduction in a subgroup of patients with 'intractable' chronic migraine $(p<0.01) ; 59.5 \%$ of patients had a $30 \%$ reduction in headache days and/or pain intensity and $47.8 \%$ had a $50 \%$ reduction (41).

In order to test whether unblinding due to local paresthesias may influence outcome in ONS trials, suprathreshold, subthreshold and no stimulation were compared in a small randomised cross-over study of eight patients suffering from chronic migraine. The suprathreshold stimulation was found to be more effective than the subthreshold one, but the latter was also superior to no stimulation (42).

A meta-analysis of the available RCTs and seven case series (total of 517 patients) suggests a superiority of real ONS over sham stimulation in chronic migraine patients, but the average effect size is modest and seems to be smaller than that found in cluster headache (43).

The effect of combined ONS and SON stimulation (SNS) was assessed retrospectively in an open study of 44 patients with chronic migraine. The combination was reported to reduce the frequency of severe headaches by $81 \%(44,45)$, with a nearly complete headache disappearance in half of the patients (45). This impressive result needs to be confirmed in a RCT. Combined ONS-SNS was found to be superior to ONS alone in a small retrospective study of chronic migraineurs (44), 


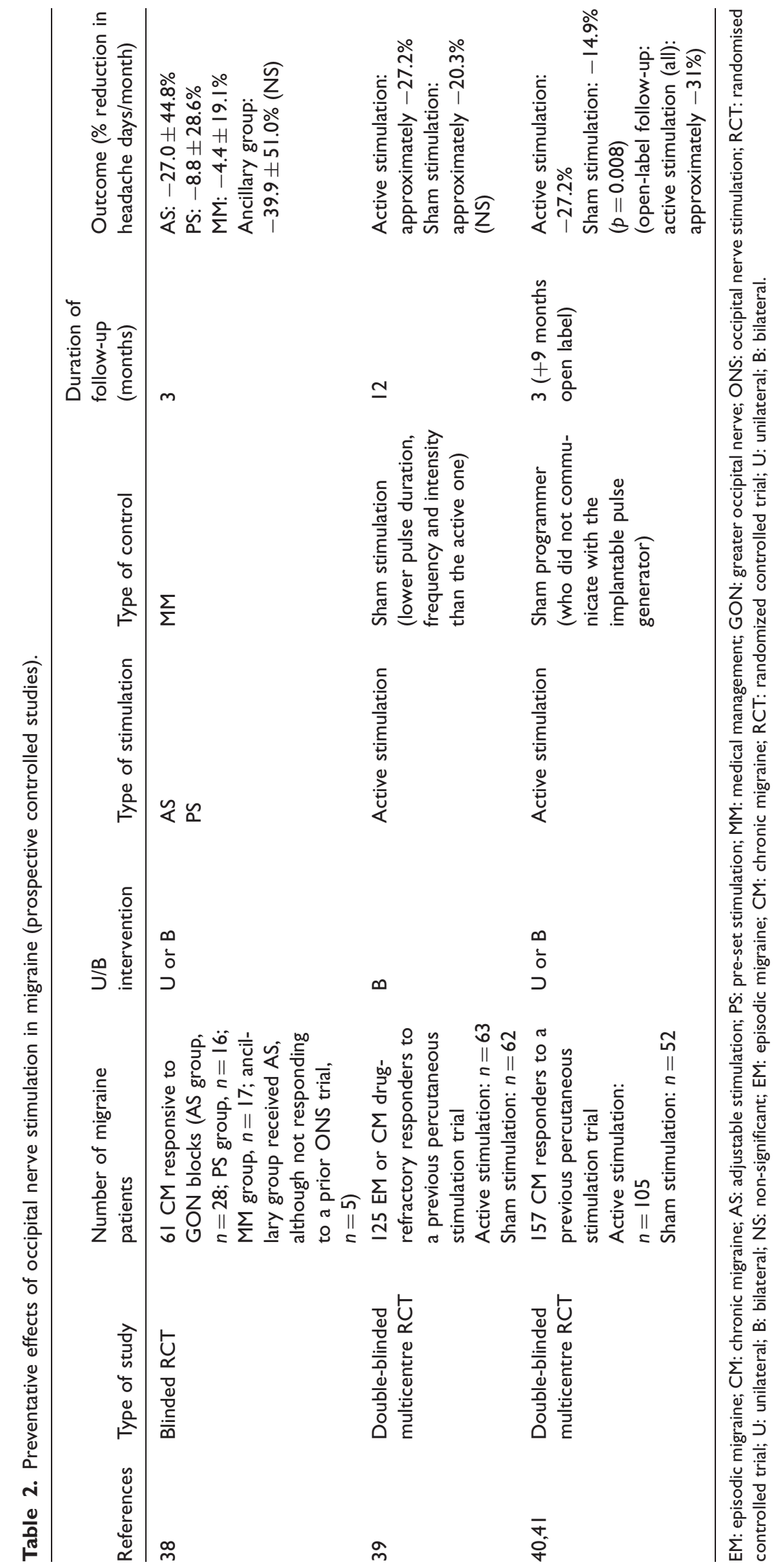


but patient selection and follow-up biases may have influenced the results (46). The combined stimulation was found to be useful in a case series of four drugresistant sporadic hemiplegic migraine patients (47). This result also needs confirmation, but it was hypothesised that PNS might inhibit cortical spreading depression (48).

\section{Cluster headache}

The evidence for the effectiveness of ONS in intractable $\mathrm{CCH}(\mathrm{iCCH})$ comes from several open-label prospective studies (49-53) and three long-term follow-up studies of the same patients (54-56). The first shamcontrolled trial (ICON) is still ongoing (57). In one trial, eight iCCH patients were implanted bilaterally: six patients reported $20-95 \%$ ameliorations of attack frequency and intensity after a follow-up of 6-27 months (49). In another pilot study, eight iCCH patients were implanted unilaterally on the attack side and prospectively followed for 3-22 months using headache diaries (50). Two patients became pain-free, three had a decrease in attack frequency of approximately $90 \%$ and two had a decrease in attack frequency of approximately $40 \%$. Overall attack intensity decreased by $50 \%$. With ONS, all of the patients could reduce preventative medications, but not interrupt them completely. Transient side shift of attacks occurred only in two patients. In both studies, a clinical benefit occurred after a delay ranging from several weeks to several months postsurgery.

In a multicentre prospective study involving 13 iCCH patients who were implanted bilaterally and followed for 3-34 months, ten patients reported an amelioration of attack intensity within a few days of the surgery (51). Among the seven patients with a followup of 12 months, two were headache-free, four had $a \geq 50 \%$ reduction of attack frequency and one had a $30 \%$ reduction of attack frequency. At the long-term follow-up, the treatment response was lost in two out of these seven patients. In a small study of three patients, two had a $>50 \%$ reduction in headache frequency already at 1 month post-surgery, whereas the last one responded after 3 months (52).

A trial with bilateral ONS including ten $\mathrm{iCCH}$ patients showed beneficial effects in nine subjects, with a mean overall improvement over time of $44 \%$ (range: $20-90 \%$ ) in terms of attack frequency; eight patients also reported a decreased intensity of any remaining attacks. ONS became effective on average at 20 days after surgery (53).

Three of these trials were subsequently extended to other patients and longer follow-up $(49,50,53)$. In the study by Burns et al., nine out of 14 patients were followed up for more than 12 months (54). Four of them reported sustained $a \geq 50 \%$ improvement of their headache. Magis et al., after long-term follow-up (range: 11-64 months), reported complete remission of attacks in eight patients out of 13 who had a follow-up exceeding 12 months, and about $90 \%$ amelioration in two patients, but most patients continued preventative drugs and the intensity of residual attacks was not significantly reduced (55). Among the 17 patients who were followed for at least 12 months during ONS, Mueller et al. demonstrated that 15 showed a $\geq 50 \%$ amelioration (56).

Some case reports of unilateral ONS-treated iCCH patients with moderate to high benefits were also published $(58,59)$. In one young patient, ONS was combined with SNS and infraorbital nerve stimulation, which was reported to produce a dramatic improvement for up to 3 years (60).

SNS alone (three patients) or combined with infraorbital neurostimulation (one patient) was found to be effective in a small retrospective study (61).

\section{Comments}

There is strong evidence from uncontrolled studies that ONS is effective for patients suffering from drug-resistant $\mathrm{CCH}$, and the effect size is greater than in chronic migraine, in which the moderate advantages from ONS are not confirmed in all RCTs. A $\geq 50 \%$ decrease in attack frequency is reported in $44-88 \%$ of $\mathrm{iCCH}$ patients, compared to $39-48 \%$ in chronic migraine. In a long-term follow-up retrospective study, $80 \%$ of $\mathrm{iCCH}$ patients reported experiencing a benefit from ONS compared to $42 \%$ of refractory chronic migraine patients (62).

The effect of ONS in iCCH is no more than symptomatic, however, since the majority of patients are unable to stop taking preventative drugs and attacks recur after interruption of the stimulation (e.g. when the battery goes flat). There is no known predictive factor for ONS efficacy; response to GON blocks (see above) does not predict response to ONS $(50,58,63)$. Nonetheless, medication overuse may be associated with a less favourable outcome after ONS (64).

Adverse effects are frequent in ONS-treated patients. The most prevalent are local pain and/or intolerable paresthesias, infections, electrode displacement and battery replacements. The latter cannot be considered an adverse effect per se, but may further increase the cost of ONS, which is estimated at $€ 28,000$ per patient (56). In one study, $58 \%$ of patients required at least one lead revision (62). Because of the paresthesias produced by the stimulation, true blinding is difficult to achieve in clinical trials, and it is thus challenging to establish the 
placebo effect with confidence. As mentioned before, however, the superiority of subthreshold ONS over no stimulation in chronic migraine studies and the rapid recurrence of attacks after cessation of the stimulation in cluster headache trials suggest that the clinical benefit is due to a genuine effect of ONS and not to a placebo effect or to the natural history of the disease $(50,63)$.

The precise mode of action of ONS needs to be determined. In a fludeoxyglucose positron emission tomography study of $\mathrm{iCCH}$ patients treated with ONS for more than 6 months, the only difference between responders and non-responders was increased metabolism in the subgenual anterior cingulate gyrus, suggesting that ONS modulates central pain control centres (65).

Taken together, the available evidence suggests that invasive ONS could be a treatment option for drugresistant $\mathrm{iCCH}$ patients. Future studies should try to determine its advantages and disadvantages with respect to sphenopalatine ganglion stimulation (see Lainez, this issue) and with respect to the upcoming non-invasive transcutaneous and transcranial neurostimulation methods (see Schoenen et al., this issue). Based on published studies, the evidence favouring ONS in chronic migraine is less convincing, and further studies are clearly needed in order to identify the predictors of response. According to the position statement of the European Headache Federation, ONS is advisable in $\mathrm{iCCH}$; in refractory chronic migraine patients, it is only considered to be acceptable, although this is based on limited evidence (66).

\section{Surgical decompressions for migraine}

As mentioned in the introduction, there is scarce evidence that nervous structures in the pericranium or face play a primary causative role in headache pathogenesis, although pain felt on the surface of the head or neck tends to be attributed merely to referred pain from the visceral part of the ophthalmic nerve (see above). Nonetheless, surgical procedures to treat headache acting directly on cranial nerves have been used for a long time.

In the last 15 years, decompression of pericranial nerves by sectioning adjacent muscles and sections of superficial nerve branches was repeatedly proposed as a possible treatment for migraine patients (67-79). Up to now, this procedure has not been attempted for other primary headaches. In the first retrospective study, resection of the corrugator supercilii muscle for rejuvenation was reported to improve migraine patients (67). It was followed by a prospective study in which the intervention was performed in headache patients with hypertrophy of the corrugator supercilii muscle who had at least $50 \%$ amelioration after one injection of $25 \mathrm{U}$ botulinum toxin type A into this muscle (68). Disappearance of headaches was reported in $66.7 \%$ of subjects, and partial improvement was reported in the remaining $33.3 \%$. The latter also underwent a transection of the zygomaticotemporal branch of the trigeminal nerve and repositioning of the temple soft tissues. After follow-up (222-494 days), all patients except one had supplementary improvement. In a subsequent unblinded prospective study, patients were allocated, depending on the origin of pain and botulinum toxin effect, to one or a combination of four surgical procedures: removal of corrugator supercilii, depressor supercilii and procerus muscles (90\% of patients); endoscopic removal of $3 \mathrm{~cm}$ of the zygomaticotemporal branch of the trigeminal nerve $(80 \%)$; resection of the semispinalis capitis muscle and shielding of the GON $(38 \%)$; and septoplasty and inferior and/or middle turbinectomies $(70 \%)$. Outcomes were compared to a nonoperated control group after a 1-year follow-up (69). In the operated group, $92 \%$ of patients were reported to have a $\geq 50 \%$ reduction in migraine headache frequency and duration, while only $15.8 \%$ of controls improved. At the end of a 5-year follow-up period, $88 \%$ of patients, $69.5 \%$ of whom were operated on at three to four sides, experienced beneficial effects from the surgical treatment, according to the authors (70).

The only sham-controlled study included 75 patients with migraine with or without aura, who reported a socalled "trigger site" (i.e. a predominant site "where the migraine headache begins and settles and corresponds to the anatomical zone of potential irritation of the trigeminal nerve") and had at least 50\% amelioration after injection of $25 \mathrm{U}$ botulinum toxin into the "trigger" area (71). They were operated on according to their "trigger sites" ("frontal", "temporal" or "occipital") and followed for 1 year. In each group, a third of patients underwent a sham operation. In the verum arm $(n=49), 83.7 \%$ of subjects from the three groups demonstrated significant amelioration or elimination of headaches, whereas in the sham arm $(n=26)$, $57.7 \%$ had a similar positive outcome.

Several retrospective studies reported possible predictors of favourable outcome (72-74) or benefit from additional surgical procedures such as supraorbital foraminotomy (75) or ligation of the occipital artery (76).

Some other retrospective studies of the effects of surgery in the frontal area in migraine are available. In the largest one using resection of corrugator and depressor muscles, $58.3 \%$ of 60 chronic or episodic migraineurs had $a \geq 50 \%$ reduction in headache days at the 6-month follow-up (77). Another study reported $a \geq 50 \%$ amelioration of headaches in 16 out of 18 
migraine patients responding to botulinum toxin and operated on at multiple sites in various combinations (78). Improvement after corrugator muscle resection was also reported in nine out of ten patients who suffered from frontally located "chronic daily headache" and improved by $\geq 50 \%$ after at least two frontal injections of botulinum toxin (79).

\section{Comments}

The proposed rationale for surgical decompression of pericranial nerves in migraine is that compression of these nerves induces inflammation and peptide release, which may reach the meninges and hence trigger headaches. This rationale may gain support from recent studies demonstrating extracranial projections of meningeal afferents (4).

With one exception, however, the evidence for the effectiveness of peripheral nerve decompressions in migraine stems mostly from retrospective or prospective uncontrolled studies. A critical point is that the migraine patients included in these studies all had some uncommon clinical features (80), such as strictly side-locked unilateral headache and the presence of "trigger sites" that are much more typical of cranial neuralgias and tension-type headaches than of migraine (81). Similarly, some additional symptoms that have been used to determine the site of the interventions are commonly found in other primary or secondary headaches such as neuralgias, headaches attributed to temporomandibular dysfunction, chronic tension-type headaches, chronic/recurring rhinosinusitis, mucosal contact points or concha bullosa and whiplash. All of these causes of head or facial pain can aggravate migraine and sometimes mimic it. An intervention that is able to influence them has the potential to improve migraine indirectly.

In addition, the strategy of selecting for surgery patients who respond to botulinum toxin type A injections at "trigger sites" is rather puzzling and has no scientific basis. In fact, most patients who were included had episodic migraine and were reported to respond to botulinum toxin in proportions ranging from $58 \%$ to $90 \%(68,69,71,79)$, which clearly contrasts with RCTs of multiple pericranial and facial injections of botulinum toxin that show no difference with saline injections (82). Hence, it is likely that many patients were selected for surgery on the basis of a placebo response. This is supported by the very high placebo response of $58 \%$ found in the only sham-controlled study of peripheral nerve decompression. Without scientific evidence, the authors hypothesize that this placebo response may be due to the fact that the incision and the undermining of pericranial tissues may have altered neurosensory functions and that some patients may have exaggerated their preoperative symptoms in order to increase their chances of being selected for surgery $(68,69,71,79)$. It is well established, however, that the placebo response in headaches is greater with invasive procedures than with drug treatments (83).

Given these confounding factors and methodological uncertainties, surgical decompression of peripheral nerves cannot be considered at the present time as an established treatment for migraine. It remains to be determined in future sham-controlled trials whether such procedures may have a lasting clinical benefit that exceeds a placebo effect in clinical subgroups of selected patients.

\section{Conclusions}

Invasive interventions on pericranial nerve branches have been used for a long time to treat headaches. Although current knowledge on primary headache pathophysiology favours a central nervous system dysfunction (84), such interventions could modulate the neuronal circuits that are involved in central sensitisation and pain control, merely producing a symptomatic non-specific effect. Among primary headaches, cluster headache appears to respond best to both GON blocks and peripheral neuromodulation, but the treatment responses are not necessarily correlated. In migraine, both procedures are only marginally effective, but this may improve in the future with advances in patient selection and treatment protocols.

Larger better-designed and comparative trials are needed in order to evaluate the long-term effects of the invasive implantation of neurostimulators or nerve decompressions. Effective blinding is a major challenge in such trials, as will be the comparison with the upcoming non-invasive neurostimulation methods.

\section{Literature search methods}

English-language publications were searched for in PubMed up to July 2015.

The following search terms were used: "migraine neurostimulation", "cluster headache neurostimulation", "migraine neuromodulation", "cluster headache neuromodulation", "migraine injection", "cluster headache injection", "migraine nerve infiltrations", "cluster headache nerve infiltrations", "migraine nerve blocks", "cluster headache nerve blocks", "migraine surgery", "cluster headache surgery" and "migraine decompression". All of the identified publications were individually assessed according to their relevance to the topic. Specific exclusion criteria included publications on single case reports and 
editorials and other review articles unless of exceptional importance. The reference lists of identified publications were also scrutinised for further relevant publications.

\section{Clinical implications}

- Suboccipital infiltrations (or greater occipital nerve blocks) are effective, evidence-based, safe and inexpensive treatments for short-term prophylaxis in cluster headache patients; they may also have some effect in selected migraine patients, particularly those with fixed unilateral headaches and ipsilateral autonomic symptoms.

- Percutaneous occipital nerve stimulation (ONS) has long-term efficacy in refractory chronic cluster headache, but it has frequent, though reversible, adverse effects, and a sham-controlled trial is not yet available. By contrast, the evidence that ONS is effective in chronic migraine is weak, although some randomised controlled trials (RCTs) indicate that it might be beneficial in subgroups of patients.

- Surgical decompression of pericranial nerves in migraine patients was reported to be superior to sham surgery in one study, and most case series are non-controlled and published by the same group. The heterogeneity of included patients, selection bias and questionable inclusion criteria are major shortcomings of these studies and do not allow us to rule out the notion that the greater part of the reported benefits is due to a placebo effect. Further better-designed RCTs are needed before surgical decompressions can be recommended in the treatment of selected migraine patients.

\section{Declaration of conflicting interests}

The authors declared the following potential conflicts of interest with respect to the research, authorship, and/or publication of this article: A Ambrosini has no conflicts of interest. J Schoenen is a consultant for Cefaly-Technology, St. Jude Medical, ATI, Medtronic, AMGEN and Gedeon Richter.

\section{Funding}

The authors received no financial support for the research, authorship, and/or publication of this article.

\section{References}

1. Irimia P, Palma JA, Fernandez-Torron R, et al. Refractory migraine in a headache clinic population. $B M C$ Neurol 2011; 11: 94.

2. Whyte CA and Tepper SJ. Adverse effects of medications commonly used in the treatment of migraine. Expert Rev Neurother 2009; 9: 1379-1391.

3. Edvinsson L, Villalón CM and MaassenVanDenBrink A. Basic mechanisms of migraine and its acute treatment. Pharmacol Ther 2012; 136: 319-333.

4. Schueler M, Neuhuber WL, De Col R, et al. Innervation of rat and human dura mater and pericranial tissues in the parieto-temporal region by meningeal afferents. Headache 2014; 54: 996-1009.

5. Schueler M, Messlinger K, Dux M, et al. Extracranial projections of meningeal afferents and their impact on meningeal nociception and headache. Pain 2013; 154: $1622-1631$.

6. Knight G. Surgical treatment of migraine. Proc $R$ Soc Med 1962; 55: 172-176.

7. Matharu MS, Boes CJ and Goadsby PJ. Management of trigeminal autonomic cephalgias and hemicrania continua. Drugs 2003; 63: 1637-1677.
8. Anthony $\mathrm{M}$. The role of the occipital nerve in unilateral headache. In: Rose FC (ed.). Current Problems in Neurology: 4. Advances in Headache Research. London: John Libbey, 1987, pp.257-262.

9. Kerr FWL. A mechanism to account for frontal headache in cases of posterior fossa tumour. J Neurosurg 1961; 18 : 605-609.

10. Ashkenazi A, Matro R, Shaw JW, et al. Greater occipital nerve block using local anaesthetics alone or with triamcinolone for transformed migraine: a randomised comparative study. J Neurol Neurosurg Psychiatry 2008; 79: 415-417.

11. Dilli E, Halker R, Vargas B, et al. Occipital nerve block for the short-term preventive treatment of migraine: a randomized, double-blinded, placebo-controlled study. Cephalalgia 2015; 35: 959-968.

12. Kashipazha D, Nakhostin-Mortazavi A, Mohammadianinejad SE, et al. Preventive effect of greater occipital nerve block on severity and frequency of migraine headache. Glob J Health Sci 2014; 6: 209-213.

13. Inan LE, Inan N, Karadas $\mathrm{O}$, et al. Greater occipital nerve for the treatment of chronic migraine: a randomized, multicenter, double-blind, parallel and placebocontrolled study. Acta Neurol Scand 2015; doi: 10.1111/ ane.12393 [Epub ahead of print].

14. Palamar D, Uluduz D, Saip S, et al. Ultrasound-guided greater occipital nerve block: an efficient technique in chronic refractory migraine without aura? Pain Physician 2015; 18: 153-162.

15. Gelfand AA, Reider AC and Goadsby PJ. Outcomes of greater occipital nerve injections in pediatric patients with chronic primary headache disorders. Pediatr Neurol 2014; 50: 135-139.

16. Afridi SK, Shields KG, Bhola R, et al. Greater occipital nerve injection in primary headache syndromes - prolonged effects from a single injection. Pain 2006; 122: 126-129. 
17. Weibelt S, Andress-Rothrock D, King W, et al. Suboccipital nerve blocks for suppression of chronic migraine: safety, efficacy, and predictors of outcome. Headache 2010; 50: 1041-1044.

18. Tobin JA and Flitman SS. Occipital nerve blocks: effect of symptomatic medication: overuse and headache type on failure rate. Headache 2009; 49: 1479-1485.

19. Kinfe T, Schuss P and Vatter H. Occipital nerve block prior to occipital nerve stimulation for refractory chronic migraine and chronic cluster headache: myth or prediction? Cephalalgia 2015; 35: 359-362.

20. Bovim G and Sand T. Cervicogenic headache, migraine without aura and tension-type headache. Diagnostic blockade of greater occipital and supra-orbital nerves. Pain 1992; 51: 43-48.

21. Ashkenazi A and Young WB. The effects of greater occipital nerve block and trigger point injection on brush allodynia and pain in migraine. Headache 2005; 45: 350-354.

22. Young W, Cook B, Malik S, et al. The first 5 minutes after greater occipital nerve block. Headache 2008; 48: 1126-1128.

23. Baron EP, Tepper SJ, Mays M, et al. Acute treatment of basilar-type migraine with greater occipital nerve blockade. Headache 2010; 50: 1057-1059.

24. Caputi CA and Firetto V. Therapeutic blockade of greater occipital and supraorbital nerves in migraine patients. Headache 1997; 37: 174-179.

25. Ilhan Alp S and Alp R. Supraorbital and infraorbital nerve blockade in migraine patients: results of 6-month clinical follow-up. Eur Rev Med Pharmacol Sci 2013; 17: 1778-1781.

26. Cady RK, Saper J, Dexter K, et al. Long-term efficacy of a double-blind, placebo-controlled, randomized study for repetitive sphenopalatine blockade with bupivacaine vs saline with the $\mathrm{Tx} 360^{\circledR}$ device for treatment of chronic migraine. Headache 2015; 55: 529-542.

27. Leroux E and Ducros A. Occipital injections for trigemino-autonomic cephalalgias: evidence and uncertainties. Curr Pain Headache Rep 2013; 17: 325.

28. Anthony M. Headache and the greater occipital nerve. Clin Neurol Neurosurg 1992; 94: 297-301.

29. Ambrosini A, Vandenheede M, Rossi P, et al. Suboccipital injection with a mixture of rapidand long-acting steroids in cluster headache: a double-blind placebo-controlled study. Pain 2005; 118 : 92-96.

30. Busch V, Jakob W, Juergens T, et al. Occipital nerve blockade in chronic cluster headache patients and functional connectivity between trigeminal and occipital nerves. Cephalalgia 2007; 27: 1206-1214.

31. Leroux E, Valade D, Taifas I, et al. Suboccipital steroid injections for transitional treatment of patients with more than 2 cluster headache attacks per day: a randomized, double-blind, placebo-controlled trial. Lancet Neurol 2011; 10: 891-897.

32. Robbins MS. Peripheral nerve blocks, steroid injections and their niche in headache medicine. Cephalalgia 2015; 25: $850-852$.
33. Lambru G, Lagrata S and Matharu MS. Cutaneous atrophy and alopecia after greater occipital nerve injection using triamcinolone. Headache 2012; 52: 1596-1599.

34. Melzack R and Wall PD. Pain mechanisms: a new theory. Science 1965; 150: 971-979.

35. DeSantana JM, Da Silva LF, De Resende MA, et al. Transcutaneous electrical nerve stimulation at both high and low frequencies activates ventrolateral periaqueductal grey to decrease mechanical hyperalgesia in arthritic rats. Neuroscience 2009; 163: 1233-1241.

36. Bartsch $\mathrm{T}$ and Goadsby $\mathrm{PJ}$. Increased responses in trigeminocervical nociceptive neurons to cervical input after stimulation of the dura mater. Brain 2003; 126: 1801-1813.

37. Serra G and Marchioretto F. Occipital nerve stimulation for chronic migraine: a randomized trial. Pain Physician 2012; 15: 245-253.

38. Saper JR, Dodick DW, Silberstein SD, et al. Occipital nerve stimulation for the treatment of intractable chronic migraine headache: ONSTIM feasibility study. Cephalalgia 2011; 31: 271-285.

39. Lipton R, Goadsby P, Cady R, et al. PRISM study: occipital nerve stimulation for treatment-refractory migraine. Cephalalgia 2009; 29(Suppl. 1): 30.

40. Silberstein S, Dodick D, Saper J, et al. Safety and efficacy of peripheral nerve stimulation of the occipital nerves for the management of chronic migraine: Results from a randomized, multicenter, double-blinded, controlled study. Cephalalgia 2012; 32: 1165-1179.

41. Dodick DW, Silberstein SD, Reed KL, et al. Safety and efficacy of peripheral nerve stimulation of the occipital nerves for the management of chronic migraine: longterm results from a randomized, multicenter, doubleblinded, controlled study. Cephalalgia 2015; 35: 344-358.

42. Slotty PJ, Bara G, Kowatz L, et al. Occipital nerve stimulation for chronic migraine: a randomized trial on subthreshold stimulation. Cephalalgia 2015; 35: 73-78.

43. Chen YF, Bramley G, Unwin G, et al. Occipital nerve stimulation for chronic migraine - a systematic review and meta-analysis. PLOS ONE 2015; 10(3): e0116786.

44. Reed KL, Black SB, Banta CJ 2nd, et al. Combined occipital and supraorbital neurostimulation for the treatment of chronic migraine headaches: initial experience. Cephalalgia 2010; 30: 260-271.

45. Reed KL, Will KR, Chapman J, et al. Combined occipital and supraorbital neurostimulation for chronic migraine headaches: an extended case series. Cephalalgia 2011; 31(Suppl. 1): 98.

46. Burns B. 'Dual' occipital and supraorbital nerve stimulation for primary headache. Cephalalgia 2010; 30: 257-259.

47. Reed KL, Will KR, Conidi F, et al. Concordant occipital and supraorbital neurostimulation therapy for hemiplegic migraine; initial experience; a case series. Neuromodulation 2015; 18: 297-303.

48. Kovacs S, Peeters R, De Ridder D, et al. Central effects of occipital nerve electrical stimulation studied by functional magnetic resonance imaging. Neuromodulation 2011; 14: 46-55. 
49. Burns B, Watkins L and Goadsby PJ. Treatment of medically intractable cluster headache by occipital nerve stimulation: long-term follow-up of eight patients. Lancet 2007; 369: 1099-1106.

50. Magis D, Allena M, Bolla M, et al. Occipital nerve stimulation for drug-resistant chronic cluster headache: a prospective pilot study. Lancet Neurol 2007; 6: 314-321.

51. Fontaine D, Sol JC, Raoul S, et al. Treatment of refractory chronic cluster headache by chronic occipital nerve stimulation. Cephalalgia 2011; 31: 1101-1105.

52. Strand NH, Trentman TL, Vargas BB, et al. Occipital nerve stimulation with the Bion ${ }^{\circledR}$ microstimulator for the treatment of medically refractory chronic cluster headache. Pain Physician 2011; 14: 435-440.

53. Mueller OM, Gaul C, Katsarava Z, et al. Occipital nerve stimulation for the treatment of chronic cluster headache - lessons learned from 18 months experience. Cent Eur Neurosurg 2011; 72: 84-89.

54. Burns B, Watkins L and Goadsby PJ. Treatment of intractable chronic cluster headache by occipital nerve stimulation in 14 patients. Neurology 2009; 72: 341-345.

55. Magis D, Gerardy PY, Remacle JM, et al. Sustained effectiveness of occipital nerve stimulation in drug-resistant chronic cluster headache. Headache 2011; 51: 1191-1201.

56. Mueller O, Diener HC, Dammann P, et al. Occipital nerve stimulation for intractable chronic cluster headache or migraine: a critical analysis of direct treatment costs and complications. Cephalalgia 2013; 33: 1283-1291.

57. Wilbrink LA, Teernstra OP, Haan J, et al. Occipital nerve stimulation in medically intractable, chronic cluster headache. The ICON study: rationale and protocol of a randomised trial. Cephalalgia 2013; 33: 1238-1247.

58. Schwedt T, Dodick D, Hentz J, et al. Occipital nerve stimulation for chronic headache - long-term safety and efficacy. Cephalalgia 2007; 27: 153-157.

59. Schwedt TJ, Dodick DW, Trentman TL, et al. Occipital nerve stimulation for chronic cluster headache and hemicrania continua: pain relief and persistence of autonomic features. Cephalalgia 2006; 26: 1025-1027.

60. Mammis A, Gudesblatt M and Mogilner AY. Peripheral neurostimulation for the treatment of refractory cluster headache, long-term follow-up: case report. Neuromodulation 2011; 14: 432-435. discussion 435.

61. Vaisman J, Markley H, Ordia J, et al. The treatment of medically intractable trigeminal autonomic cephalalgia with supraorbital/supratrochlear stimulation: a retrospective case series. Neuromodulation 2012; 15 : 374-380.

62. Brewer AC, Trentman TL, Ivancic MG, et al. Long-term outcome in occipital nerve stimulation patients with medically intractable primary headache disorders. Neuromodulation 2013; 16: 557-562. discussion 563-564.

63. Oh MY, Ortega J, Bellotte JB, et al. Peripheral nerve stimulation for the treatment of occipital neuralgia and transformed migraine using a C1-2-3 subcutaneous paddle style electrode: a technical report. Neuromodulation 2004; 7: 103-112.

64. Paemeleire K, Van Buyten JP, Van Buynder M, et al. Phenotype of patients responsive to occipital nerve stimulation for refractory head pain. Cephalalgia 2010; 30: $662-673$.

65. Magis D, Bruno MA, Fumal A, et al. Central modulation in cluster headache patients treated with occipital nerve stimulation: an FDG-PET study. BMC Neurol 2011; 11: 25.

66. Martelletti P, Jensen RH, Antal A, et al European Headache Federation. Neuromodulation of chronic headaches: position statement from the European Headache Federation. J Headache Pain 2013; 14: 86.

67. Guyuron B, Varghai A, Michelow BJ, et al. Corrugator supercilii muscle resection and migraine headaches. Plast Reconstr Surg 2000; 106: 429-434.

68. Guyuron B, Tucker T and Davis J. Surgical treatment of migraine headaches. Plast Reconstr Surg 2002; 109: 2183-2189.

69. Guyuron B, Kriegler JS, Davis J, et al. Comprehensive surgical treatment of migraine headaches. Plast Reconstr Surg 2005; 115: 1-9.

70. Guyuron B, Kriegler JS, Davis J, et al. Five-year outcome of surgical treatment of migraine headaches. Plast Reconstr Surg 2011; 127: 603-608.

71. Guyuron B, Reed D, Kriegler JS, et al. A placebo-controlled surgical trial of the treatment of migraine headaches. Plast Reconstr Surg 2009; 124: 461-468.

72. Larson K, Lee M, Davis J, et al. Factors contributing to migraine headache surgery failure and success. Plast Reconstr Surg 2011; 128: 1069-1075.

73. Liu MT, Armijo BS and Guyuron B. A comparison of outcome of surgical treatment of migraine headaches using a constellation of symptoms versus botulinum toxin type A to identify the trigger sites. Plast Reconstr Surg 2012; 129: 413-419.

74. Lee M, Monson MA, Liu MT, et al. Positive botulinum toxin type a response is a prognosticator for migraine surgery success. Plast Reconstr Surg 2013; 131: 751-757.

75. Chepla KJ, Oh E and Guyuron B. Clinical outcomes following supraorbital foraminotomy for treatment of frontal migraine headache. Plast Reconstr Surg 2012; 129: 656e-662e.

76. Chmielewski L, Liu MT and Guyuron B. The role of occipital artery resection in the surgical treatment of occipital migraine headaches. Plast Reconstr Surg 2013; 131: 351e-356e.

77. Dirnberger F and Becker K. Surgical treatment of migraine headaches by corrugator muscle resection. Plast Reconstr Surg 2004; 114: 652-657.

78. Poggi JT, Grizzell BE and Helmer SD. Confirmation of surgical decompression to relieve migraine headaches. Plast Reconstr Surg 2008; 122: 115-122.

79. de Ru JA, Schellekens PP and Lohuis PJ. Corrugator supercilii transection for headache emanating from the frontal region: a clinical evaluation of ten patients. J Neural Transm 2011; 118: 1571-1574.

80. D'Amico D, Leone M and Bussone G. Side-locked unilaterality and pain localization in long-lasting headaches: migraine, tension-type headache, and cervicogenic headache. Headache 1994; 34: 526-530.

81. Headache Classification Committee of the International Headache Society (IHS). The International Classification 
of Headache Disorders, 3rd edition (beta version). Cephalalgia 2013; 33: 629-808.

82. Jackson JL, Kuriyama A and Hayashino Y. Botulinum toxin A for prophylactic treatment of migraine and tension headaches in adults: a meta-analysis. JAMA 2012; 307: 1736-1745.
83. Meissner K, Fassler M, Rucker G, et al. Differential effectiveness of placebo treatments: a systematic review of migraine prophylaxis. JAMA Intern Med 2013; 173: 1941-1951.

84. De Tommaso M, Ambrosini A, Brighina F, et al. Altered sensory processing in migraine. Nat Rev Neurol 2014; 10 : 144-155. 